\title{
Modelo de aproximación lineal para la medición de resiliencia en cadenas de suministro
}

\section{Linear approximation model for the assessment of resilience in a supply chain}

\author{
Daniel Romero Rodríguez ${ }^{1} \quad$ Weimar Ardila Rueda ${ }^{1 *} \quad$ Ernesto Cantillo Guerrero ${ }^{1}$ \\ Alvaro Sierra Altamiranda ${ }^{1} \quad$ Fabián Sánchez Sánchez ${ }^{1}$
}

Recibido 15 de septiembre de 2015, aceptado 21 de marzo de 2016

Received: September 15, 2015 Accepted: March 21, 2016

\begin{abstract}
RESUMEN
La identificación del nivel de resiliencia de un sistema es fundamental para la toma de decisiones en la prevención y planeación de estrategias de recuperación ante fallas que lo puedan afectar. Este artículo busca diseñar una métrica de aproximación lineal para la medición de resiliencia en cadenas de suministro ante eventos disruptivos inesperados. Las métricas tradicionales de resiliencia simplifican el comportamiento de un sistema después de la ocurrencia de una falla, dificultando que las mediciones se puedan realizar en escenarios de fallas de mayor complejidad. Una métrica general de resiliencia es desarrollada y validada en un caso simulado de una cadena de suministro de dos eslabones con interrupciones en el proceso de transporte. Los resultados confirman que las métricas de resiliencia tradicionales sobreestiman los niveles de resiliencia del sistema, debido a la inhabilidad de modelar diferentes escenarios de eventos disruptivos. Los resultados del caso simulado sugieren que la nueva métrica mejora la estimación de resiliencia en comparación con las métricas lineales previas y, adicionalmente provee la flexibilidad necesaria para ser utilizada en otros tipos de sistema diferentes a cadenas de suministro.
\end{abstract}

Palabras clave: Métricas de resiliencia, cuantificar resiliencia, sistemas resilientes, administración de riesgos en cadenas de suministro, interrupciones en la cadena de suministro.

\section{ABSTRACT}

The following research aims to the design of a linear metric approximation for the measurement of supply chain resilience under unexpected events. The identification of the system's resilience level is a key step for the decision making in the prevention and planning of recovery strategies, in the case of a disruptive failure. Traditional measurements of resilience are based on strong simplifications of the evolution of systems' performance, which leads to an inaccurate analysis of more complex scenarios. A general metric is developed and is validated on a simulated case of a two-echelon supply chain with transportation disruptions. Results proved that previous linear metrics over-estimated the resilience level due to the inability to model different disruptions scenarios. The results suggest that the new approach improved the former linear metric, and provided the required flexibility to model resilience for different systems besides supply chains.

Keywords: Resilience metrics, quantifying resilience, resilient systems, supply chain risk management, supply chain disruptions.

\footnotetext{
1 Ingeniería Industrial. Universidad Autónoma del Caribe. Calle 90 \#46-112. Barranquilla, Colombia. E-mail: daniel.romero37@uac.edu.co; Weimar.ardila@uac.edu.co, ecantillo@uac.edu.co; alvaro.sierra10@uac.edu.co; fsanchez@uac.edu.co

* Autor de correspondencia
} 


\section{INTRODUCCIÓN}

En el contexto de cadenas de suministro, los eventos que pueden generar interrupciones están aumentando en número y frecuencia, afectando la capacidad de estas para cumplir con la entrega de productos o prestación de servicios. Lo anterior evidencia la necesidad de cadenas de suministros resilientes capaces de responder de manera adecuada a eventos que puedan perturbar su desempeño [1]. La resiliencia de un sistema se define como la capacidad de absorber un evento disruptivo y recuperar el estado original o deseado del sistema [2].

La resiliencia en cadenas de suministros se define como la capacidad adaptativa que tienen las cadenas de suministros para prepararse frente a la ocurrencia de eventos inesperados, responder a interrupciones, y recuperarse de estos manteniendo continuidad en las operaciones a un nivel deseado de conectividad y control sobre la estructura y operaciones [3]. Una cadena de suministro resiliente continuará funcionando con la menor pérdida económica posible aumentando su competitividad en comparación con otros sistemas que no se encuentren preparados ante eventos disruptivos [4].

La valoración de la resiliencia en una cadena de suministro se ha realizado principalmente con encuestas y entrevistas a tomadores de decisiones, lo que tiene un error potencial debido a que las respuestas pueden tener sesgos por el entendimiento imperfecto del sistema por parte de los actores involucrados en las decisiones [5]. Otra dificultad de la medición basada en encuestas es la imposibilidad de distinguir entre interrupción generada por un evento o el de una falla por múltiples eventos.

La medición de resiliencia busca cuantificar la capacidad de un sistema para absorber y recuperarse ante la adversidad. De tal manera que en el nivel deseado de resiliencia se logra un balance entre las vulnerabilidades y las capacidades del sistema [5]. En la actualidad existen indicadores de resiliencia en otras áreas del conocimiento que se pueden adaptar al contexto de las cadenas de suministro, sin embargo, es necesario introducir factores de ajuste a los indicadores que garanticen la flexibilidad requerida para la medición de resiliencia en otros dominios.
El artículo está organizado de la siguiente manera: en primera instancia se presenta una discusión de las métricas propuestas en la literatura para una aproximación lineal de la resiliencia de un sistema. En segunda instancia se propone una métrica discreta lineal para medir resiliencia, la que posteriormente es validada por medio de un caso simulado de una cadena de suministro con interrupción del proceso de transporte. Finalmente se analiza la importancia de la métrica propuesta al generalizar la medición de diferentes escenarios.

\section{ESTADO DEL ARTE}

La revisión de la literatura de los conceptos de resiliencia y métricas de resiliencia, permite dimensionar el alto grado de difusión de estos conceptos en diferentes áreas del conocimiento, como las ciencias sociales y aplicadas. En la presente revisión se delimita la literatura a mediciones de resiliencia implementadas en áreas de las ciencias aplicadas, en especial de la ingeniería, donde sobresalen estudios de resiliencia en infraestructura crítica, organizaciones, logística humanitaria y transporte.

En el estudio de la resiliencia en cadenas de suministro un alto porcentaje de la literatura se ha enfocado en las estrategias que se deben adoptar para prevenir y enfrentar eventos disruptivos [5].

En este sentido se destacan las siguientes estrategias de recuperación:

i) Redundancia: Disponer de capacidad adicional de equipos, personal e inventarios [6-7].

ii) Flexibilidad: La capacidad de ajustarse a cambios del mercado en forma rápida [8-9].

Por otra parte, en cuanto a las estrategias que buscan la prevención de fallas se resaltan las siguientes:

i) Diseño de la red de suministro: Planear la cadena de suministro con confiabilidad y resiliencia, es decir, que se minimice la probabilidad de ocurrencia de algún evento disruptivo y, en caso de que ocurra algún incidente, el diseño del sistema ayude a la recuperación inmediata [10-12].

ii) Colaboración: Alinear estrategias y compartir información con los miembros de la cadena de 
suministro para planear conjuntamente como prevenir y/o reaccionar ante diferentes eventos disruptivos [13-14].

iii) Diversificación: Consiste en eliminar las dependencias del sistema, es decir, evitar depender de un solo producto o proveedor, se requiere diversificar las ventas, operaciones y el abastecimiento [15-16].

La medición de resiliencia parte de la necesidad de cuantificar el estado actual del sistema para establecer un plan de acción para protegerlo de interrupciones causadas por eventos inesperados como desastres naturales, fallas en maquinaria, entre otros. El desarrollo de una métrica de resiliencia está sujeto a la identificación de las dimensiones del concepto de resiliencia, entre las más comunes se encuentran: robustez, redundancia, recursividad, rapidez, capacidad de absorber, y adaptabilidad [17-18]. El diseño de una métrica que refleje todas las dimensiones resulta una tarea compleja por la naturaleza multivariada del concepto de resiliencia. Este desafío se ha estudiado mediante encuestas o herramientas de diagnóstico que miden resiliencia con base en la percepción de tomadores de decisiones o evaluadores del sistema [5, 19-20].

Las métricas que se analizan a continuación no corresponden a mediciones subjetivas del sistema basadas en diagnósticos de expertos o en las encuestas mencionadas previamente. Principalmente se discuten indicadores que se basan en la toma de datos de pérdidas del nivel de desempeño y tiempos de recuperación. Lo anterior implica que se estudian aquellos modelos que utilizan datos del sistema y reflejan la medición en un solo indicador. La discusión se centra en modelos para un solo evento disruptivo, no para aquellos escenarios con múltiples eventos disruptivos en serie [20].

En la Figura 1 se observa el "triángulo de la resiliencia", a partir del que se formula una métrica en la que se mide la pérdida de desempeño del sistema y el tiempo de recuperación para calcular el desempeño promedio de este último, desde la ocurrencia del evento disruptivo hasta su completa recuperación [17]. La métrica asociada al concepto del triángulo de resiliencia es considerada una de las métricas más utilizadas en la actualidad [21].

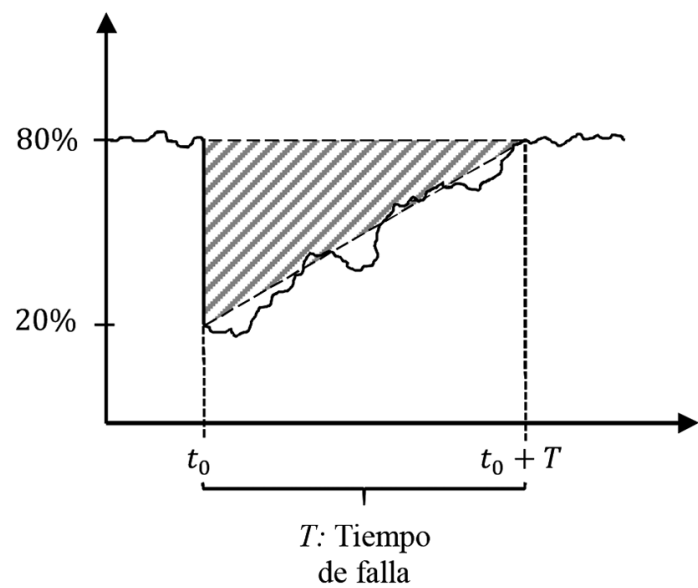

Figura 1. Triángulo de la resiliencia.

La estimación teórica del nivel de desempeño promedio se realiza por medio de la siguiente ecuación:

$Q(t)$

$T_{O E}$ : $\quad$ Tiempo de ocurrencia del evento

$T_{L C}$ : Tiempo de control del estudio

$$
R=\int_{T_{O E}}^{T_{O E}+T_{L C}} \frac{Q(t)}{T_{L C}} d t
$$

La métrica de la ecuación (1) tiene como dificultad que requiere encontrar la función matemática del desempeño del sistema con respecto al tiempo [22], lo que no es una opción viable siempre en el contexto de cadenas de suministro, por esta razón se desarrolló una métrica análoga de carácter lineal y discreta para estimar el triángulo de resiliencia, orientada a tomadores decisiones prácticos [23].

\section{X: Pérdida del nivel de desempeño \\ T: Tiempo de recuperación \\ $T^{*}$ : Tiempo de control del estudio}

$$
R(X, T)=1-\frac{X T}{2 T^{*}}
$$

En la misma orientación del cálculo del desempeño promedio se tiene la siguiente métrica utilizada en el contexto de redes de transporte, en donde se optimiza la toma de decisiones en la etapa de prevención y en la etapa posterior a la ocurrencia del evento [24-26]: 
$d_{w}$ : Demanda después del desastre

$D_{w}$ : Demanda previa al desastre

$$
R=\frac{E\left(\Sigma_{w \in W} d_{w}\right)}{\Sigma_{w \in W} D_{w}}
$$

En otros estudios se proponen métricas que consisten en la multiplicación de diferentes propiedades de la resiliencia de un sistema, de esta manera capturan las dimensiones de capacidad de absorción, velocidad de recuperación y adaptabilidad del sistema [18].

\section{$S_{p}$ : Velocidad de recuperación}

$F_{r}$ : Nivel de desempeño en estado recuperado

$F_{d}$ : Nivel de desempeño en estado interrumpido

$F_{0}$ : Nivel de desempeño en estado inicial

$$
\rho\left(S_{p}, F_{r}, F_{d}, F_{0}\right)=S_{p} \frac{F_{r}}{F_{0}} \frac{F_{d}}{F_{0}}
$$

En el esquema de multiplicación de métricas se favorece el número de dimensiones de resiliencia que se analizan, sin embargo, se dificulta el análisis y comparación de la medición, a diferencia de las métricas previas donde el área del triángulo de resiliencia tiene una mayor facilidad en la interpretación de resultados.

En otra línea de medición de resiliencia se definen los estados del sistema antes, durante y después de un evento disruptivo, con el fin elaborar un perfil más amplio de un sistema con una modelación del proceso de interrupción y recuperación ajustados en una mayor proporción a la realidad [27-31].

Bajo el esquema de los estados del sistema se define una métrica general, en donde se utiliza la

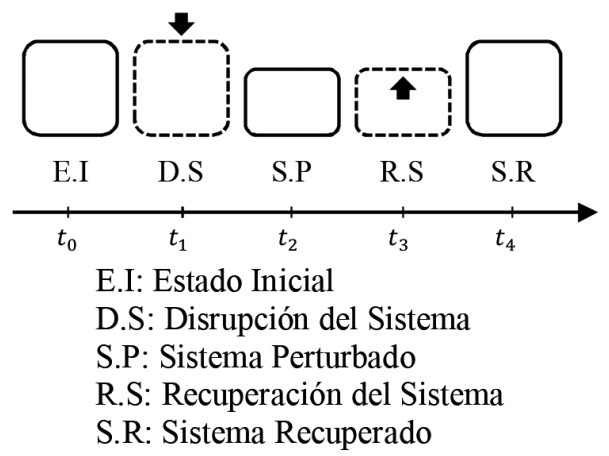

Figura 2. Estados del sistema. recuperación del nivel de desempeño hasta un tiempo $t$, y el total de pérdida máximo del desempeño [15]:

$$
R=\frac{\text { Recuperación }(t)}{\text { Máxima pérdida }(t)}
$$

La ecuación (5) deriva la siguiente expresión:

$F\left(t_{r} \mid e_{j}\right):$ Nivel de desempeño en estado de recuperación $F\left(t_{d} \mid e_{j}\right)$ : Nivel de desempeño en estado interrumpido $F\left(t_{0}\right)$ : Nivel de desempeño en estado inicial

$$
R\left(t_{r} \mid e_{j}\right)=\frac{F\left(t_{r} \mid e_{j}\right)-F\left(t_{d} \mid e_{j}\right)}{F\left(t_{0}\right)-F\left(t_{d} \mid e_{j}\right)}
$$

Desde una perspectiva conceptual la ecuación (6) es débil frente a las métricas del triángulo de resiliencia, debido a que mide el porcentaje de recuperación del sistema en un tiempo $t$, brindando una valoración sesgada que no considera que la resiliencia además de ser la capacidad de recuperación de un sistema tras la ocurrencia de un evento disruptivo, también representa la capacidad de absorber y mitigar el impacto inicial.

Del análisis de las métricas de resiliencia revisadas se encuentra que las principales deficiencias son: i) la falta de generalización de las métricas a sistemas diferentes. ii) Inflexibilidad de las métricas de los escenarios modelados, se asume una caída instantánea del desempeño del sistema, y que se da inicio a la recuperación inmediatamente después de la falla.

La contribución del presente artículo se resumen en los siguientes tres aportes:

i) Desarrollo de una medición que considera cualquier variable de desempeño de un sistema, con respecto a su duración total de la falla. En esta medición, la duración total de falla se compone de tres tiempos: el tiempo de declive, el tiempo de reacción al alcanzar el mínimo desempeño (tiempo muerto), y el tiempo de recuperación o respuesta.

ii) Generalización de la medición de resiliencia, capturando diferentes escenarios de interrupción de las operaciones, de tal manera que se tenga la flexibilidad necesaria para cuantificar resiliencia en cualquier escenario lineal de declive, recuperación, y desempeño 
mínimo, e incluso en diferentes áreas del conocimiento.

iii) Obtención de una medición más exacta de la capacidad de resiliencia de un sistema, permitiendo mejorar la toma de decisiones y garantizar la sostenibilidad de un sistema ante la ocurrencia de eventos disruptivos inesperados de cualquier complejidad o magnitud.

\section{MEDICIÓN DE RESILIENCIA: MODELO DE APROXIMACIÓN LINEAL}

A partir de las debilidades detectadas en las métricas discutidas en la sección previa, a continuación se plantean, los supuestos y requerimientos para la construcción de una métrica de resiliencia.

En primera instancia se observa que en el esquema del triángulo de resiliencia se simplifica el comportamiento del impacto de una falla al asumir que la pérdida del nivel de desempeño ocurre de forma inmediata, y la recuperación del mismo inicia en ese instante. En la Figura 3 se observa el escenario simplificado.

Por otra parte, existe un escenario general, el que permite una valoración precisa de la resiliencia para casos en los que el decremento en los niveles de desempeño del sistema se da de forma progresiva por la presencia de mecanismos de contención para hacer frente a problemas asociados a la variabilidad

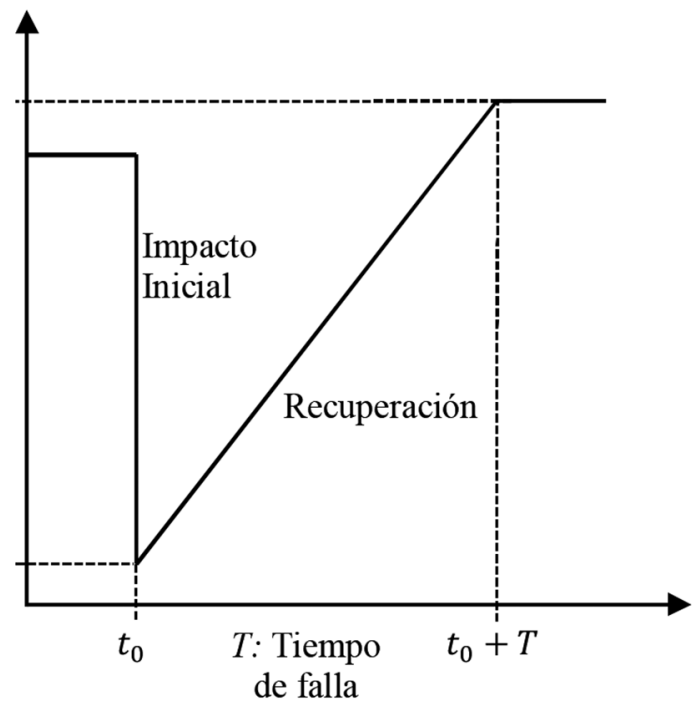

Figura 3. Caída de desempeño instantánea. natural. La recuperación no necesariamente empieza de manera inmediata posiblemente por la falta de preparación del sistema y se da de manera progresiva. Lo anterior implica que una adecuada valoración de la resiliencia de un sistema debe considerar que la duración total de la falla en el sistema es equivalente a la suma de su tiempo de declive, el tiempo de reacción del sistema al alcanzar su mínimo desempeño y el tiempo de recuperación.

\section{$t_{D}$ : Tiempo de declive \\ $t_{M}$ : Tiempo de desempeño mínimo \\ $t_{R}$ : Tiempo de recuperación}

$$
T=t_{D}+t_{M}+t_{R}
$$

A continuación se observa el perfil de una interrupción del sistema con las fases identificadas con el supuesto de linealidad en las diferentes fases posteriores al evento disruptivo.

En la Figura 4 se observa el comportamiento del nivel de desempeño del sistema en el escenario general [15].

A partir del marco teórico desarrollado para el triángulo de resiliencia y el análisis de la Figura 3,

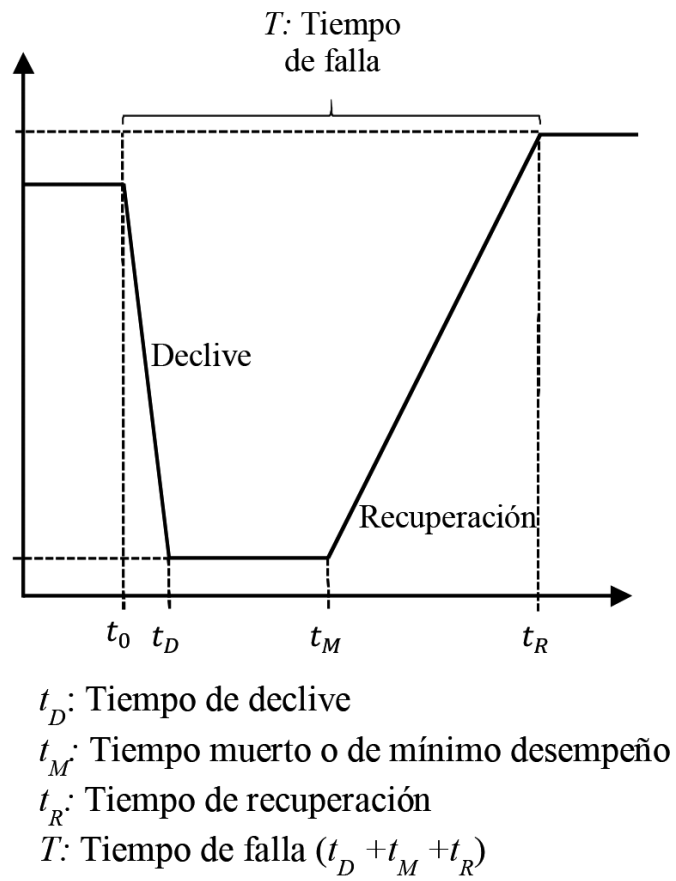

Figura 4. Caso general de falla en el sistema. 
se propone la siguiente métrica general para la valoración de la resiliencia de un sistema:

$$
\begin{array}{r}
R=1-\frac{\frac{x t_{D}}{2}+x t_{M}+\frac{x t_{R}}{2}}{T^{*}} \\
R=1-\frac{x}{2 T^{*}}\left(t_{D}+2 t_{M}+t_{R}\right)
\end{array}
$$

La ecuación (9), a diferencia de las expuestas previamente, considera el tiempo de declive del sistema y el tiempo de desempeño mínimo.

Al reemplazar la ecuación (7) en la ecuación (9) se obtiene la siguiente expresión:

$$
R=1-\frac{x}{2 T^{*}}\left(T+t_{M}\right)
$$

Donde $T$ es el tiempo total desde la falla en el sistema hasta que el sistema se ha recuperado.

La principal ventaja de esta métrica es su generalidad, y capacidad de brindar una adecuada valoración de la resiliencia en cualquier escenario lineal de declive, recuperación, y desempeño mínimo, debido a que además de considerar los tres parámetros que influyen en el tiempo de duración de la falla (tiempo de declive, tiempo muerto, tiempo de respuesta), esta fácilmente puede ajustarse al contexto de cada caso en específico, al asumir el valor de cero en aquellos en donde no sea necesario considerar cualquiera de los tres intervalos de tiempo que implica la duración de la falla.

El indicador propuesto tiene la flexibilidad para hacer aproximaciones lineales de la resiliencia asociada con cualquier variable de desempeño del sistema, como la calidad de las obras civiles e infraestructura, o el nivel de servicio de una cadena de suministros.

\section{EVALUACIÓN DE MÉTRICAS}

La validación de la métrica propuesta se desarrolla por medio de la medición de la resiliencia de una cadena de suministros multieslabón simulada. El sistema de referencia tiene dos eslabones: Fabricante y Distribuidor, quienes abastecen al cliente final. Se utiliza un modelo de simulación discreto, en donde se genera una falla de transporte que interrumpe el flujo de materiales entre el distribuidor y su cliente durante una semana.

La estructura del modelo de simulación cuenta con tres etapas. En la primera parte del modelo se concentran todos los bloques orientados a simular el flujo de información de materiales en la cadena de suministros descrita. La segunda parte busca simular la ocurrencia de la falla y con ella generar el estado falla en la cadena de suministros. Finalmente, se determina y registra el desempeño del sistema para cada réplica.

El desempeño del sistema se evalúa cada día de acuerdo con la capacidad del distribuidor de cumplir con la demanda del cliente final, i.e. mediante el nivel de servicio de la cadena de suministro.

Nivel de servicio $=\frac{\# \text { Pedidos entregados }}{\# \text { Pedidos totales }}$

Asimismo, el modelo cuenta con una serie de parámetros que le permiten simular el comportamiento de una cadena de suministros, como lo son: 1) Una política de inventario de revisión continua que define el tamaño de pedidos y punto de reorden para la generación de las órdenes de pedido, 2) el tiempo de abastecimiento o el tiempo que le toma al fabricante cumplir con el pedido del distribuidor y 3) el tiempo de falla del sistema, que para este caso es equivalente a una semana de interrupción del flujo físico entre el fabricante y el distribuidor.

Se realizaron 25 réplicas para significancia estadística y se midió la resiliencia de cada una utilizando las ecuaciones (2) y (10).

En la Figura 6 se observan cinco replicas en donde el sistema después de una falla en el transporte, mantuvo algunos días un desempeño mínimo.

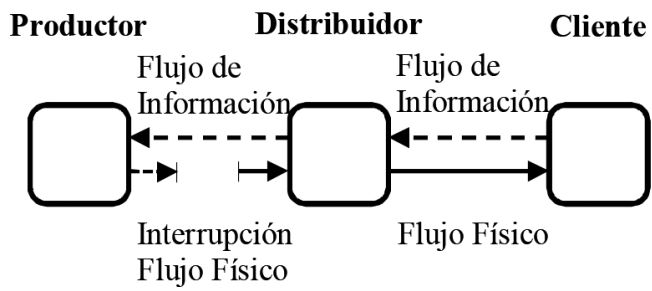

Figura 5. Estructura de la cadena de suministro simulada. 


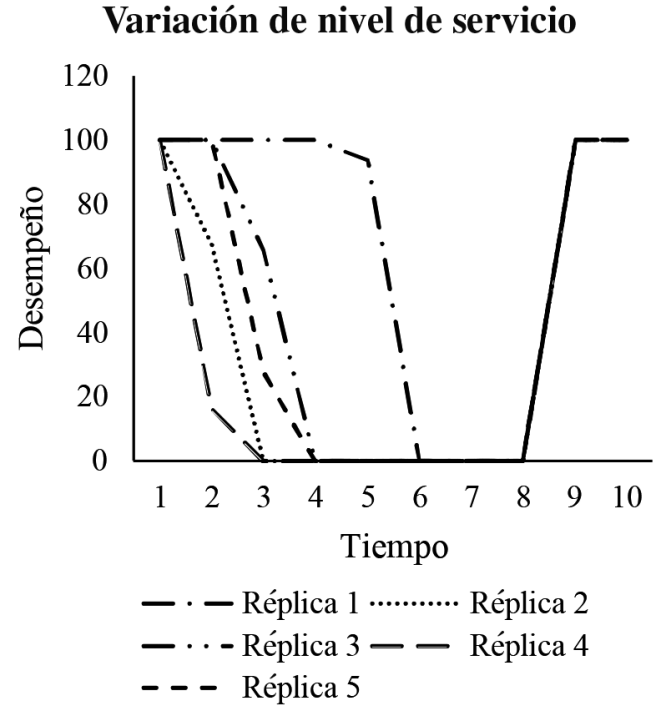

Figura 6. Instancias de fallas de la cadena de suministro.

En la Tabla 1 se muestra los resultados de la medición de resiliencia mediante la métrica de resiliencia global propuesta y la métrica de resiliencia predictiva de la ecuación (2). Asimismo, se calcula la diferencia entre los valores de ambas mediciones.

De la Tabla 1 se observa que la métrica de la ecuación (2) sobreestima el valor de la resiliencia de sistema estudiado, en comparación a la métrica propuesta de resiliencia. Las mediciones de resiliencia son iguales para ambas métricas en el caso en que el tiempo del desempeño mínimo del sistema es igual a cero. El caso anterior se presenta en las réplicas 6 y 25.

$\mathrm{Al}$ analizar los resultados de ambas mediciones se encuentra que la diferencia en el promedio es del $13 \%$, lo que representa un error en la estimación de la resiliencia del 20,31\%.

En la Figura 7 se observa que para cada réplica del modelo de simulación, la métrica propuesta es menor o igual a la valoración realizada por la ecuación (2).

La expresión que cuantifica la diferencia teórica entre la ecuación (10) y la ecuación (2) está dada por:

$$
\Delta R=\left[1-\frac{x}{2 T^{*}}\left(T+t_{M}\right)\right]-\left[1-\frac{x}{2 T^{*}}(T)\right]
$$

Tabla 1. Resultados de medición de resiliencia.

\begin{tabular}{|c|c|c|c|}
\hline$\#$ & $\begin{array}{c}\text { Medición } \\
\text { Resiliencia. } \\
\text { Ecuación (10) } \\
(\%)\end{array}$ & $\begin{array}{c}\text { Medición Resiliencia } \\
\text { de Zobel. } \\
\text { Ecuación (2) } \\
(\%)\end{array}$ & $\begin{array}{c}\text { Diferencia } \\
(\Delta \%)\end{array}$ \\
\hline 1 & 83 & 87 & -3 \\
\hline 2 & 77 & 83 & -7 \\
\hline 3 & 63 & 77 & -13 \\
\hline 4 & 77 & 83 & -7 \\
\hline 5 & 57 & 73 & -17 \\
\hline 6 & 90 & 90 & 0 \\
\hline 7 & 77 & 83 & -7 \\
\hline 8 & 63 & 77 & -13 \\
\hline 9 & 50 & 70 & -20 \\
\hline 10 & 63 & 77 & -13 \\
\hline 11 & 57 & 73 & -17 \\
\hline 12 & 50 & 70 & -20 \\
\hline 13 & 63 & 77 & -13 \\
\hline 14 & 57 & 73 & -17 \\
\hline 15 & 63 & 77 & -13 \\
\hline 16 & 63 & 77 & -13 \\
\hline 17 & 57 & 73 & -17 \\
\hline 18 & 63 & 77 & -13 \\
\hline 19 & 57 & 73 & -17 \\
\hline 20 & 57 & 73 & -17 \\
\hline 21 & 63 & 77 & -13 \\
\hline 22 & 57 & 73 & -17 \\
\hline 23 & 50 & 70 & -20 \\
\hline 24 & 57 & 73 & -17 \\
\hline 25 & 90 & 90 & 0 \\
\hline Media & 64 & 77 & -13 \\
\hline
\end{tabular}

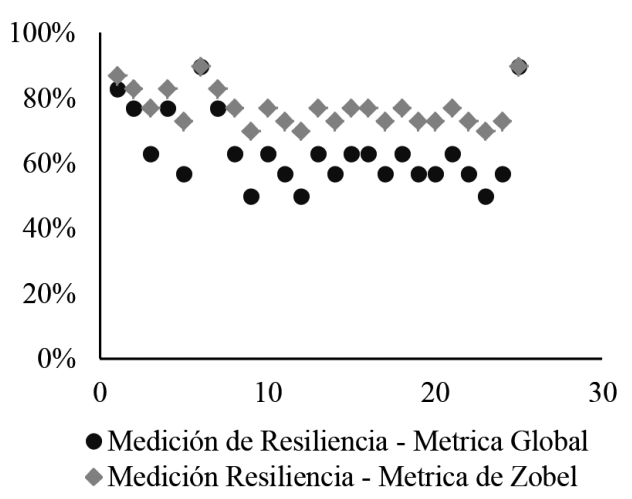

Figura 7. Resultados de medición de resiliencia.

$$
\Delta R=-\frac{x}{2 T^{*}} t_{M}
$$

La diferencia en la ecuación (13) se debe a que la ecuación (2) aproxima la curva de desempeño 
del sistema analizando el área de un triángulo, mientras que la ecuación (10) considera geometrías que se aproximan con mayor exactitud a la forma de la curva de desempeño. En aquellos escenarios en que la recuperación del sistema empieza de forma instantánea ambas métricas van a arrojar el mismo valor, debido a que el $t_{M}$ es igual a cero, lo que significa que no existe diferencia entre ambas mediciones.

Los resultados evidencian que la métrica propuesta garantiza una mayor exactitud en la aproximación lineal de la resiliencia del sistema, lo que permite una medición ajustada a la realidad del sistema.

\section{CONCLUSIONES}

Dentro de los hallazgos del estudio se destaca la definición de los escenarios de variación del desempeño de un sistema luego de la ocurrencia de un evento disruptivo. Estos escenarios permiten entender las distintas maneras en las que un sistema puede reaccionar tras la falla, dependiendo del contexto evaluado y la variable de referencia de desempeño de este sistema.

La definición de los escenarios de variación permitió detectar debilidades de la métrica del triángulo de la resiliencia, al momento de valorar la resiliencia cuando la caída en el desempeño del sistema se produce de manera progresiva. Adicionalmente se presenta una demora del sistema para reaccionar después de haber llegado al mínimo de su desempeño.

En cuanto a la medición se ha propuesto una métrica de resiliencia que no asume la caída instantánea del nivel de desempeño y, al mismo tiempo, reconoce la posible existencia de un tiempo donde el sistema tiene un desempeño mínimo. La validación de la métrica propuesta en un modelo de simulación de una cadena de suministro dio como resultado que la aproximación lineal de resiliencia mejora en un $20,13 \%$ la medición, en comparación con las métricas lineales anteriores. El resultado anterior confirma que la medición propuesta iguala o mejora la estimación de los niveles de resiliencia. Por otra parte, la métrica desarrollada tiene la capacidad de adaptarse fácilmente a las condiciones de cada uno de los escenarios de variación definidos por el tomador de decisión.
Finalmente, en cuanto a investigaciones futuras se identifica la necesidad de crear métricas de resiliencia capaces de considerar la ocurrencia de múltiples eventos dentro del mismo intervalo de tiempo de análisis. Por otra parte, se hace necesario construir métricas que no consideren el supuesto de linealidad.

\section{REFERENCIAS}

[1] H. Carvalho, A.P. Barroso, V.H. Machado, S. Azevedo and V. Cruz-Machado. "Supply chain redesign for resilience using simulation". Computers \& Industrial Engineering. Vol. $62 \mathrm{~N}^{\circ} 1$, pp. 329-341. Febrero 2012. ISSN: 0360-8352. DOI: 10.1016/j.cie.2011.10.003

[2] C. Martin and H. Peck. "Building the resilient supply chain". The international journal of logistics management. Vol. 15 $\mathrm{N}^{\mathrm{o}}$ 2. 2004. ISSN: 0957-4093. DOI: http:// dx.doi.org/10.1108/09574090410700275

[3] S.Y. Ponomarov and M.C. Holcomb. "Understanding the concept of supply chain resilience". The International Journal of Logistics Management. Vol. 20 N$^{\circ} 1$, pp. 124143. 2009. ISSN: 0957-4093.DOI: http:// dx.doi.org/10.1108/09574090910954873

[4] Y. Sheffi. "The resilient enterprise: overcoming vulnerability for competitive advantage". MIT Press Books, pp. 325. Cambridge, USA. 2005. ISBN: 9780262693493

[5] B.R. Tukamuhabwa, M. Stevenson, J.Busby and M. Zorzini. "Supply chain resilience: definition, review and theoretical foundations for further study". International Journal of Production Research. Vol. $53 \mathrm{~N}^{\mathrm{o}}$ 18, pp. $5592-$ 5623. Abril 2015. ISSN: 0020-7543. DOI: 10.1080/00207543.2015.1037934

[6] C.S. Tang. "Robust strategies for mitigating supply chain disruptions". International Journal of Logistics: Research and Applications. Vol. $9 \mathrm{~N}^{\circ}$ 1, pp. 33-45. Marzo 2006. ISSN: 1367-5567. DOI: http://dx.doi. org/10.1080/13675560500405584

[7] L. Urciuoli, S. Mohanty, J. Hintsa and E. Gerine-Boekesteijn. "The resilience of energy supply chains: a multiple case study approach on oil and gas supply chains to Europe". Supply Chain Management: An International Journal. Vol. $19 \mathrm{~N}^{\circ}$ 1, pp. 46-63. 
2014. ISSN: 1359-8546. DOI: http://dx.doi. org/10.1108/SCM-09-2012-0307

[8] T.J. Pettit, J. Fiksel and K.L. Croxton. "Ensuring supply chain resilience: development of a conceptual framework". Journal of Business Logistics. Vol. $31 \mathrm{~N}^{\circ} 1$, pp. 1-21. 2010. ISSN: 2158-1592. DOI: 10.1002/j.2158-1592.2010.tb00125.x

[9] P. Mensah and Y. Merkuryev. "Developing a Resilient Supply Chain". Procedia-Social and Behavioral Sciences. Vol. 110, pp. 309319. Enero 2014. ISSN: 1877-0428. DOI: 10.1016/j.sbspro.2013.12.875

[10] A. Azaron, K.N. Brown, S.A. Tarim, and M. Modarres. "A multi-objective stochastic programming approach for supply chain design considering risk". International Journal of Production Economics. Vol. 116 $\mathrm{N}^{\circ}$ 1, pp. 129-138. Noviembre 2008. ISSN: 0925-5273. DOI: 10.1016/j.ijpe.2008.08.002

[11] M.S. Daskin, L.V. Snyder and R.T. Berger. "Logistics systems: design and optimization". Springer, pp. 39-65. USA. 2005. ISBN: 978-0-387-24971-1

[12] L.V. Snyder, M.P. Scaparra, M.S. Daskin and R.L. Church. "Tutorials in operations research". INFORMS, pp. 234-257. USA. 2006. ISBN: 13978-1-877640-20-9. DOI: http://dx.doi.org/10.1287/educ.1063.0025

[13] U. Jüttner and S. Maklan. "Supply chain resilience in the global financial crisis: an empirical study". Supply Chain Management: An International Journal. Vol. $16 \mathrm{~N}^{\circ}$ 4, pp. 246-259. 2011. ISSN: 1359-8546. DOI: http://dx.doi. org/10.1108/13598541111139062

[14] P.R. Kleindorfer and G.H. Saad. "Managing disruption risks in supply chains". Production and operations management. Vol. $14 \mathrm{~N}^{\circ} 1$, pp. 53-68. Marzo 2005. ISSN: 1937-5956. DOI: 10.1111/j.19375956.2005.tb00009.x

[15] S. Park, T.E. Lee and C.S. Sung. "A threelevel supply chain network design model with risk-pooling and lead times". Transportation Research Part E: Logistics and Transportation Review. Vol. $46 \mathrm{~N}^{\circ}$ 5, pp. 563-581. Septiembre 2010. ISSN: 1366-5545 DOI:10.1016/j. tre.2009.12.004

[16] V. Babich, A.N. Burnetas and P.H. Ritchken. "Competition and diversification effects in supply chains with supplier default risk".
Manufacturing \& Service Operations Management. Vol. $9 \mathrm{~N}^{\circ}$ 2, pp. 123-146. Abril 2007. ISSN: 1523-4614. DOI:http://dx.doi. org/10.1287/msom.1060.0122

[17] M. Bruneau, S.E. Chang, R.T. Eguchi, G.C. Lee, T.D. O'Rourke, A.M. Reinhorn, M. Shinozuka, K. Tierney, W.A. Wallace and D.V. Winterfeldt. "A framework to quantitatively assess and enhance the seismic resilience of communities". Earthquake spectra. Vol. 19 $\mathrm{N}^{\circ}$ 4, pp. 733-752. Noviembre 2003. ISSN: 8755-2930. DOI: 10.1193/1.1623497

[18] R. Francis and B. Bekera. "A metric and frameworks for resilience analysis of engineered and infrastructure systems". Reliability Engineering \& System Safety. Vol. 12, pp. 90-103. Enero 2014. ISSN: 0951-8320. DOI: 10.1016/j.ress.2013.07.004

[19] U. Soni, V. Jain and S. Kumar. "Measuring supply chain resilience using a deterministic modeling approach". Computers \& Industrial Engineering. Vol. 74, pp. 11-25. Agosto 2014. ISSN: 0360-8352. DOI:10.1016/j. cie.2014.04.019

[20] C.W. Zobel and L. Khansa. "Characterizing multi-event disaster resilience". Computers \& Operations Research. Vol. 42, pp. 83-94. Febrero 2014. ISSN: 0305-0548. DOI: 10.1016/j.cor.2011.09.024

[21] M. Xu, X. Wang and L. Zhao. "Predicted supply chain resilience based on structural evolution against random supply disruptions". International Journal of Systems Science: Operations \& Logistics. Vol. $1 \mathrm{~N}^{\circ} 2$, pp. 105117. Julio 2014. ISSN: 2330-2674. DOI: 10.1080/23302674.2014.934748

[22] G.P. Cimellaro, A.M. Reinhorn and M. Bruneau. "Framework for analytical quantification of disaster resilience". Engineering Structures. Vol. $32 \mathrm{~N}^{\circ} 11$, pp. 3639-3649. Noviembre 2010. ISSN: 01410296. DOI: 10.1016/j.engstruct.2010.08.008

[23] C.W.Zobel. "Representing perceived tradeoffs in defining disaster resilience". Decision Support Systems. Vol. $50 \mathrm{~N}^{\mathrm{o}}$ 2, pp. 394403. Enero 2011. ISSN: 0167-9236. DOI: 10.1016/j.dss.2010.10.001

[24] L. Chen and E. Miller-Hooks. "Resilience: an indicator of recovery capability in intermodal freight transport". Transportation Science. Vol. 46 N $^{\circ}$ 1, pp. 109-123. Febrero 
2012. ISSN: 0041-165. DOI: http://dx.doi. org/10.1287/trsc. 1110.0376

[25] E. Miller-Hooks, X. Zhang and R. Faturechi. "Measuring and maximizing resilience of freight transportation networks". Computers \& Operations Research. Vol. $39 \mathrm{~N}^{\circ} 7$, pp. 1633-1643. Julio 2012. ISSN: 03050548. DOI: 10.1016/j.cor.2011.09.017

[26] X. Zhang, E. Miller-Hooks, and K. Denny. "Assessing the role of network topology in transportation network resilience". Journal of Transport Geography. Vol. 46, pp. 35-45. Junio 2015. ISSN: 0966-6923. DOI: $10.1016 /$ j.jtrangeo.2015.05.006

[27] D. Henry and J.E. Ramirez-Marquez. "Generic metrics and quantitative approaches for system resilience as a function of time". Reliability Engineering \& System Safety. Vol. 99, pp. 114-122. Marzo 2012. ISSN: 0951-8320 DOI: 10.1016/j.ress.2011.09.002

[28] K. Barker, J.E. Ramirez-Marquez and C.M. Rocco. "Resilience-based network component importance measures". Reliability
Engineering \& System Safety. Vol. 117, pp. 89-97. Septiembre 2013. ISSN: 09518320 DOI: 10.1016/j.ress.2013.03.012

[29] R. Pant, K. Barker, J.E. Ramírez-Marquez and C.M. Rocco. "Stochastic measures of resilience and their application to container terminals". Computers \& Industrial Engineering. Vol. 70, pp. 183-194. Abril 2014. ISSN: 0360-8352. DOI: 10.1016/j. cie.2014.01.017

[30] H. Baroud, K. Barker and J.E. RamirezMarquez. "Importance measures for inland waterway network resilience". Transportation research part E: logistics and transportation review. Vol. 62, pp. 55-67. Febrero 2014. ISSN: 1366-5545. DOI: 10.1016/j.tre.2013.11.010

[31] H. Baroud, J.E. Ramirez-Marquez, K. Barker and C.M. Rocco. "Stochastic measures of network resilience: Applications to waterway commodity Flows". Risk Analysis. Vol. 34 $\mathrm{N}^{\mathrm{o}}$ 7, pp. 1317-1335. Febrero 2014. ISSN: 1539-6924. DOI: 10.1111/risa.12175 